\title{
Review Article \\ Genomic Resources and Tools for Gene Function Analysis in Potato
}

\author{
Glenn J. Bryan and Ingo Hein \\ Genetics Programme, Scottish Crop Research Institute, Invergowrie, Dundee DD2 5DA, UK
}

Correspondence should be addressed to Glenn J. Bryan, glenn.bryan@scri.ac.uk

Received 22 November 2007; Revised 26 August 2008; Accepted 28 September 2008

Recommended by Pushpendra Gupta

\begin{abstract}
Potato, a highly heterozygous tetraploid, is undergoing an exciting phase of genomics resource development. The potato research community has established extensive genomic resources, such as large expressed sequence tag (EST) data collections, microarrays and other expression profiling platforms, and large-insert genomic libraries. Moreover, potato will now benefit from a global potato physical mapping effort, which is serving as the underlying resource for a full potato genome sequencing project, now well underway. These tools and resources are having a major impact on potato breeding and genetics. The genome sequence will provide an invaluable comparative genomics resource for cross-referencing to the other Solanaceae, notably tomato, whose sequence is also being determined. Most importantly perhaps, a potato genome sequence will pave the way for the functional analysis of the large numbers of potato genes that await discovery. Potato, being easily transformable, is highly amenable to the investigation of gene function by biotechnological approaches. Recent advances in the development of Virus Induced Gene Silencing (VIGS) and related methods will facilitate rapid progress in the analysis of gene function in this important crop.
\end{abstract}

Copyright (c) 2008 G. J. Bryan and I. Hein. This is an open access article distributed under the Creative Commons Attribution License, which permits unrestricted use, distribution, and reproduction in any medium, provided the original work is properly cited.

\section{INTRODUCTION}

Cultivated potato, the world's third most important human food crop, is a tetraploid outbreeder and suffers acutely from inbreeding depression. Genetic mapping is generally performed at the diploid level, using highly heterozygous clones as parents, and several diploid maps of potato have been generated [1], including one of the densest plant genetic maps [2]. Considerable progress has also been made in working at the tetraploid level $[3,4]$. These efforts have led to the development of large numbers of molecular markers of all of the main types, which in some cases allow comparison of different potato maps or between potato and the closely related tomato. Genetic mapping has also led to knowledge of locations of many potato genes, notably those conferring resistance to many of the pests and pathogens that present a threat to potato [5] and genes influencing tuber traits [6]. Despite these advances, the lack of described mutational variation for potato is a disadvantage of its outbreeding mating habit, and renders genetic complementation problematic for the majority of genes. However, potato is relatively easy to transform, and so technologies such as overexpression and antisense technology are options for investigating gene function. Results of such experiments are not always so easy to interpret, and improved methods for functional analysis are critical to the future of potato breeding and genetics.

This article provides an overview of genomics resources currently available for potato, and the likely future developments in this area, paying particular emphasis to tools being developed for investigating gene function.

\section{BASIC FACTS ABOUT THE POTATO GENOME}

Cultivated potato has a chromosome number of $2 n=4 x$ $=48$, and a haploid genome size of $\sim 850 \mathrm{Mb}$, roughly six times that of Arabidopsis thaliana and twice the size of the rice genome [7]. Although small chromosome size has been a limitation for cytogenetic analysis in potato, notable advances have been made using pachytene chromosomes and extended DNA "fibres" for fluorescence in situ hybridization (FISH) [8]. The potato genome is very similar in size to its close relative tomato, and genetic maps of the two species show high levels of macrocolinearity [9]. 
Information on how well the two genomes are conserved at the microsyntenic level should start to become available as outputs from the respective genome projects accumulate. The tomato genome mainly comprises low-copy-number sequences, which diverged rapidly in evolutionary time [10]. Schweizer et al. [11], who characterised the potato genome in terms of the amounts of different classes of repetitive DNA, suggest that the more highly repeated sequences comprise only $4-7 \%$ of the potato genome, suggesting that it was relatively devoid of highly repetitive DNA sequences, thus supporting the earlier tomato study. It is also known that the majority of tomato heterochromatin is found in centromeric regions with almost all of the euchromatic DNA located distally in long uninterrupted tracts, a structural feature likely to be true of potato [12]. Gene isolation and recent BAC-end sequencing efforts are providing the first detailed glimpses of the genome structure in potato. Using BAC-end sequence and full BAC sequence data, it has also been shown that potato $(34 \%)$ contains considerably less repetitive DNA than tomato $(46 \%)$, this difference being consistent with relative genome sizes of the two crops ( 850 versus $1000 \mathrm{Mb}$, resp.) [13].

\section{STRUCTURAL GENOMICS RESOURCES FOR POTATO}

\subsection{EST resources}

The generation of large expressed sequence tag (EST) collections is a primary route for large-scale gene discovery. There have been several efforts to generate EST resources for potato [14-16]. The potato gene index (http:// compbio.dfci.harvard.edu/tgi/cgi-bin/tgi/gimain.pl?) contains almost 220000 ESTs, assembled into more than 30000 "contigs" with over 26000 singletons. These efforts, while not exhaustive, comprise a major genomics resource for potato researchers, perhaps comprising between $50-70 \%$ of the total potato gene "repertoire." These ESTs will form an important source not only for the discovery of candidate genes and genetic markers, but also for the development of microarrays, until the whole genome sequence becomes available in potato. For instance, EST data from a number of different genotypes are also a rich source for the discovery of single nucleotide polymorphism (SNP) and simple sequence repeat (SSR) markers. For example, Tang et al. [17] demonstrate how large numbers of "eSNPs" can be mined from EST data using an SNP discovery pipeline (QualitySNP).

\subsection{Large-insert genomic libraries and physical maps}

Bacterial artificial chromosome (BAC) libraries have become the main vehicle for performing map-based gene cloning and physical mapping in potato. Several BAC libraries have been constructed from cultivated potato [18] and some of its wild relatives, for example, the diploids Solanum bulbocastanum [19], Solanum pinnatisectum [20], and the Mexican hexaploid Solanum demissum [21]. These libraries represent a potentially useful resource for the study of comparative genome organisation and evolution in potato and the wider Solanaceae. A BAC library has been constructed from the male parent (RH89-039-16) of the cross used to make the ultra-high-density (UHD) genetic map of potato with 10000 loci [2], and is being used for construction of a genomewide potato physical map. Other significant developments arising from the use of these BAC libraries include the use of BAC clones and fluorescence in situ hybridization (FISH) to develop chromosome-specific cytogenetic DNA markers for chromosome identification in potato [22].

\section{GENE ISOLATION IN POTATO}

\subsection{Map-based approach}

Mapping efforts in potato have also led to the generation of knowledge concerning the genetic architecture of a number of characters, including pest and disease resistance, tuber quality traits, dormancy, tuber shape, and colour. Also, several potato genes have been isolated using a map-based approach $[18,23,24]$, with most of these aimed at isolation of major genes for resistance to the more serious pests and pathogens of potato, the late blight pathogen Phytophthora infestans (Mont. de Bary), potato cyst nematodes (PCN), and potato virus $\mathrm{X}$ (PVX). These activities have necessitated the development of dense genetic maps around the target resistance loci, as well as concomitant generation of genomic resources, such as BAC libraries. These gene cloning efforts have afforded early glimpses into the structure of the potato genome, through the sequencing of a considerable number of large-insert clones. For instance, a study of Gpa2/Rx1 resistance gene "cluster" provided important information concerning the evolution and structure of $R$ gene loci and has shown beyond any doubt that resistances to different pests/pathogens can be coded by structurally similar genes from the same gene cluster.

The R3 locus, which maps to a cluster of genes for resistance against $P$. infestans and other resistance genes on the short arm of chromosome XI, has shown to comprise two very tightly linked resistance genes $(R 3 a$ and $R 3 b)$ with distinct specificities against $P$. infestans [25]. The $R 3$ locus was found to be syntenic with the $I 2$ locus of tomato, and a comparative approach was used to isolate $R 3 a$, which is constitutively expressed along with some of its paralogous genes [26]. It is highly likely that the same approach will allow the future isolation of other $P$. infestans resistance genes on the same chromosome. Similarly, there are now determined efforts to isolate genes from late blight resistance "hotspots" on other potato chromosomes. A notable example is the recent work on potato chromosome IV, whereby several resistance genes against $P$. infestans map to the same locus [27-29].

These are but a few of several successful map-based gene isolation efforts, but these illustrate how comparative genomics, either between different potato genotypes or between different Solanaceous plant species, can be used as a tool for accelerating the normally laborious task of gene isolation, and they bode well for the future of Solanaceae genomic research. As knowledge of the genome structure of potato and tomato increases, the isolation of such genes should become more facile. 


\subsection{Candidate gene approach}

A candidate gene approach has also been used for isolating plant genes that underlie specific traits [30]. In potato, cloning of the gene Gro1-4, which confers resistance to pathotype Rol of the cyst nematode Globodera rostochiensis, has been achieved using a joint candidate gene/mapping approach [31]. The gene was found to colocalise in a large segregating population with a marker derived from a "resistance-gene-like" sequence. The marker was used to isolate 15 members of a closely related gene family from genomic libraries. By taking into account all available information (inheritance patterns in resistant and susceptible germplasm, mapping data, DNA sequence information), it was possible to reduce the number of candidates to three genes, which were subsequently tested for complementation of a susceptible phenotype by stable transformation. The identified functional gene, a member of the TIR-NBS-LRR class, differs from susceptible members of the same family by 29 amino acid changes. This approach may be used in future for isolation of other resistance genes/QTLs conferring partial and durable resistance to the major potato pests and pathogens.

Another example of the use of candidate gene approach in potato is the isolation of $P$ gene that encodes anthocyanin biosynthetic enzyme flavonoid $3^{\prime}, 5^{\prime}$-hydroxylase $\left(\mathrm{f} 3^{\prime} 5^{\prime} \mathrm{h}\right)$, and is responsible for the production of blue/purple anthocyanin pigments in tissues like tubers, flowers, or stems [32]. In this study, a Petunia $\mathrm{f}^{\prime} 5^{\prime} \mathrm{h}$ gene was used to screen a potato cDNA library prepared from purple-coloured flowers and stems. Six positively hybridizing cDNA clones were sequenced and all appeared to be derived from a single gene that shared $85 \%$ sequence identity at the amino acid level with Petunia $\mathrm{f3}^{\prime} 5^{\prime} \mathrm{h}$. The potato gene cosegregated with purple tuber colour in a diploid population and was found to be expressed in tuber skin only in the presence of the anthocyanin regulatory locus $\mathrm{I}$. One of the $\mathrm{f} 3^{\prime} 5^{\prime} \mathrm{h}$ cDNA clone that was placed under the control of a doubled CaMV 35S promoter was also used for transformation of the red-skinned cultivar "Desiree." Tuber and stem tissues that were coloured red in Desiree were purple in nine of 17 independently transformed lines, confirming the hypothesis that the transformed gene corresponded to the P locus.

In another study, DNA sequence variation was analysed at the invGE/GF locus (duplicate invertase genes $I n v G E$ and InvGF) on potato chromosome IX which colocalizes with a cold-sweetening QTL [33]. The study focused on 188 tetraploid potato cultivars, which were assessed for chip quality and tuber starch content. Two closely correlated invertase alleles, invGE- $f$ and invGF- $d$, were associated with better chip quality in three breeding populations, and one allele (invGF-b) was associated with lower tuber starch content. The potato invGE gene was also found to be orthologous to the tomato invertase gene $\operatorname{Lin} 5$, causal for a fruit-sugar-yield QTL. These results suggested that natural variation for sugar yield in tomato fruits and that for sugar content in potato tubers are controlled by functional variants of orthologous invertase genes.
These few examples clearly demonstrate the potential of using the candidate gene approach in potato. It is also clear that the extensive knowledge of tuber biochemistry and the large number of potato gene sequences should enable its further application for tuber quality traits.

\section{POTATO GENOME SEQUENCING}

The ultra-high-density (UHD) genetic map of potato [2] forms the underlying framework for construction of a genome-wide physical map of the potato genome. Physical map construction is being carried out in two phases. First, approximately 73000 clones from a BAC-library have been fingerprinted using a nonselective AFLP-based method. The fingerprint data has been used to assemble the RH BACs into roughly $7000 \mathrm{BAC}$ contigs, with a similar number of "singletons" (i.e., single BAC clones). The second phase entails anchoring of the contigs and single BACs to the UHD map using a BAC pooling method, which should also reduce the number of contigs and increase the average contig size. Subsequent contiging will use a reduced stringency alignment approach which will reduce the number of contigs still further. The integrated genetic and physical map will be the main platform, which will be used for obtaining the DNA sequence of the potato genome. It is expected that approximately 1800 contigs will be anchored to the genetical map, and these scaffolds will be the starting point for genome sequencing. A BAC-end sequence resource, comprising more than 140000 reads, has also been generated for the project [13]. The ongoing tomato and potato sequencing projects will have huge implications for those working in the Solanaceae, and will further sharpen the requirement for functional genomics tools.

\section{ANALYSIS OF POTATO GENE EXPRESSION}

A wide range of gene expression technologies have been used by potato researchers. Expression analysis is a discipline that is still very much in transition and it is likely to undergo significant development in the future, notably with recent developments in "next generation" sequencing (NGS) technologies, which have the potential to radically change the way gene discovery is performed.

\subsection{CDNA-AFLP}

The cDNA-AFLP technique has been used to study gene expression from stolon formation to sprouting in a range of different tissues during the potato tuber life cycle $[34,35]$. Approximately 18000 transcript-derived fragments (TDFs) were observed, and over 200 "process specific" TDFs belonging to different stages of potato tuber life cycle were isolated and sequenced. The sequence similarities of these TDFs to known genes give insights into the kinds of processes occurring during tuberisation, dormancy, and sprouting. This technique is extremely sensitive and can detect differences among gene family members indistinguishable by Northern blotting. A useful advance has been the realization that a large proportion of cDNA-AFLP fragments show 
genetic polymorphism in segregating populations and can be mapped as transcriptome-derived genetic markers [36]. Importantly, these markers show less centromeric clustering than AFLP markers derived directly from genomic DNA and appear to be targeted specifically to transcriptionally active regions of the genome. This method has been used to perform a large scale survey of genes differentially expressed during the tuber life cycle, and the isolation of some of their promoter regions [37]. Many genes expressed in the tuber life cycle are involved in defence, stress, storage, and signal transduction pathways. Twelve cis-acting elements were identified, and are known to be responsive to environmental stimuli known to play an important role during the tuber life cycle (light, sugars, hormones, etc.). More recently, a potato transcription map, based on cDNA-AFLP and containing approximately 700 TDFs, has been generated [38]. One of the disadvantages of cDNA-AFLP is that it does not provide gene sequence information and requires laborious isolation of gene fragments from polyacrylamide gels for sequence characterization.

\subsection{SAGE}

Serial analysis of gene expression (SAGE), which generates short cDNA sequence tags [39, 40] using a concatemerization-based method, has been used to examine global gene expression in potato tubers, generating 58322 sequence tags (of length 19 nucleotides) of which 22233 were unique [41]. Putative functions were assigned to almost 700 of those tags occurring at least ten times and roughly $70 \%$ matched each known potato EST sequence. This technology has the advantage over microarray technology in being an "open" technology, with the possibility of discovering "new" transcripts. Rapid amplification of complementary DNA ends (RACE) cloning was used to verify the reliability of SAGE tag annotation using EST sequences from more than one cultivar. Seventy two per cent of tags represented genes that participated in a known biological process, with the largest group (43\%) consisting of transcripts active in physiological processes, about half of which were involved in metabolism. There were no transcripts found which were involved in photosynthesis. Of the 50 most abundant transcripts from the mature tuber, protease inhibitors were the dominant class, which is in good agreement with previous EST projects [14, 15].

The methodologies described briefly in this section are alternatives to the microarrays, which may ultimately be replaced by NGS methods. For example, Emrich et al. [42] recently demonstrated how such technologies can be used to extend significantly the EST resources for maize. The authors used a laser capture microdissection method to isolate rare transcripts from shoot apical meristems and then sequenced the corresponding cDNAs using 454 technology. This type of approach could be used in potato to identify transcripts not present in current EST databases or to extend the range of potato germplasm represented, currently limited to a few cultivars. All expression studies share the "problem" that they are only indicative of the function of particular genes or sets of genes in biological processes, and require functional analyses whereby the function of the candidate genes are compromised or exaggerated in some way (e.g., overexpression, silencing). This issue will be addressed in a subsequent section of this article.

\section{MICROARRAYS: TOOLS FOR HIGH-THROUGHPUT GENE EXPRESSION ANALYSIS}

\section{1. cDNA microarrays}

The available potato EST resources comprise an unknown but significant fraction of the gene complement of potato, and are derived from several genotypes, tissues, and environmental influences. A nonredundant set of 10000 of these ESTs was used by the Institute for Genomic Research (TIGR) to develop a cDNA potato microarray that was made available to the research community at minimal cost. Moreover, the same organisation offered a transcription profiling service to allow the evaluation of these arrays by a wide range of users working on different Solanaceous plant species asking different biological questions. This allowed generation of massive microarray data that is publicly available (http://www.tigr.org/tdb/potato/profiling_service2 .shtml\#AProcedure). However, this platform had the disadvantage of containing a very small proportion of the potato gene repertoire. Moreover, as the "TIGR array" was based on spotted cDNAs, it was inherently difficult to achieve a high level of reproducibility. Rensink et al. [43] have used this platform to identify genes involved in abiotic stress responses, with more than 3000 genes found to be significantly up- or downregulated in response to at least one of the stress conditions used (cold, heat, salt). In another detailed study, expression of 1315 genes during tuber development was examined, where transient changes in gene expression were found to be relatively uncommon and several new genes were found to be differentially expressed during tuber development [44]. These studies, while informative, highlight the dilemma faced by plant molecular biologists in prioritizing genes for further study from a large number of candidate genes in the absence of genetic information and mutations in target trait genes.

\subsection{Oligonucleotide microarrays}

Long oligonucleotide arrays that have been manufactured by various technology providers have also been found useful in potato since the use of short oligonucleotide arrays may lead to misinterpretations due to high degree of allelic heterozygosity in this crop. For this purpose, the potato oligo chip initiative (POCI) has selected the Agilent " $44 \mathrm{~K}$ feature platform" system, which was made available for use in 2006. This system is very flexible and allows for redesign of the array as more gene sequence information becomes available. Kloosterman et al. [45] described the design of this platform and demonstrated its utility by analyzing different stages of tuber initiation and growth. 


\section{FUNCTIONAL STUDIES IN POTATO}

Potato geneticists and breeders have generated a great deal of information about the location of genes and QTLs coding for important potato traits, including pest and disease resistance and tuber traits. The volume of gene sequence information, notably from cDNA sequencing and the genome project, will increase rapidly in the coming years. Developments in genetics and structural genomics are beginning to be matched by concomitant development of functional genomics tools. Potato has a strong need for a high-density gene map or a genome sequence, to place gene sequences in their genetic/genomic context. Relatively high-throughput methods are also needed for testing and assessing gene function. The availability of mutant populations of potato will also be of tremendous value in this regard [46]. Potato cultivars are highly heterozygous and contain very high levels of "genetic load." It has been estimated that there is one SNP approximately every $25 \mathrm{bp}$ [47]. If individual alleles can be "isolated" in the homozygous condition, there is no telling what information they would yield about potato biology. The nonavailability of mutants may largely be overcome by recourse to use of diploid self-compatible potato clones for the development of mutant populations or by mining of variant alleles in heterozygous germplasm. Functional studies currently rely on the use of transformation-based techniques or use of viral vector-mediated gene delivery systems for the establishment of information regarding gene function. There have been some recent tantalising developments in functional genetics/genomics tools and resources for potato. Of course gene expression profiling or microarray studies have a role to play in the identification of a pool of candidate genes potentially involved in any given biological process. These methods, in combination with other functional genomics tools such as RNA interference (RNAi), virus-induced gene silencing (VIGS), and activation tagged lines, have the potential to facilitate the identification of the role of thousands of potato genes over the next several years. Furthermore, combining structural genetics approaches (such as QTL and candidate gene mapping) with functional genomics information (such as microarrayderived gene expression data for candidate genes) has great potential for the dissection of many complex, polygenic potato traits.

\subsection{Virus-induced gene silencing (VIGS)}

Virus-induced gene silencing (VIGS) is a powerful tool for plant functional genomics. VIGS exploits an RNA-mediated antiviral defense mechanism in plants. This phenomenon has been exploited for gene silencing through the use of virus vectors carrying host target genes that are directed against the corresponding plant mRNAs [48]. VIGS is increasingly used to generate transient loss-of-function assays, and is a powerful reverse-genetics tool in functional genomic programs as an alternative to stable transformation. In potato, two viral vectors, potato virus $\mathrm{X}$ (PVX) and tobacco rattle virus (TRV), have been successfully utilized for VIGS $[49,50]$. Faivre-Rampant et al. [49] have shown that a binary
PVX-based vector, pGR106, [51, 52] is effective in triggering VIGS of phytoene desaturase (PDS) in both diploid and cultivated tetraploid Solanum species. In this study, silencing was maintained throughout the foliar tissues and tubers and could also be triggered and sustained in in vitro micropropagated tetraploid potato for several cycles and on in vitro generated microtubers. Similarly, PDS silencing with TRV has been observed in cultivated potato, as well as the diploid wild species S. bulbocastanum and S. okadae, and the distantly related hexaploid S. nigrum [50]. In the same study, silencing of known resistance genes (e.g., $R 1, R x$, and $R B$ ) in normally resistant plants yielded a compatible interaction in detached leaf tests. A modification of the leaf inoculation used for both PVX- and TRV-based silencing was demonstrated for TRV in a so-called "agrodrench" method, in which soil adjacent to the plant root is drenched with an Agrobacterium suspension carrying the TRV-derived VIGS vectors [53]. TRV-based silencing of genes such as PDS, a $20 \mathrm{~S}$ proteasome subunit (PB7) or Mg-protoporphyrin chelatase $(\mathrm{Chl} \mathrm{H})$ by agrodrench has been shown to be efficient for different members of the Solanaceae including Nicotiana benthamiana, tomato, pepper, tobacco, potato, and petunia.

$N$. benthamiana provides a particularly suitable model system for Solanaceae species, including potato, as it is highly amenable to manipulations such as VIGS and virus- or Agrobacterium-mediated overexpression of candidate genes (Figure 1) [54]. Indeed, many silencing studies have been conducted in $N$. benthamiana to demonstrate involvement of candidate genes involved in the plant disease resistance (including the hypersensitive response; HR), abiotic stress, cellular signaling, and secondary metabolite biosynthesis [55]. Recently, for example, Gilroy et al. [56], using a combination of VIGS and biochemical approaches, demonstrated that the cysteine protease cathepsin B is required for the HR. Silencing of cathepsin B in N. benthamiana prevented programmed cell death (PCD) and compromised disease resistance induced by Erwinia amylovora and Pseudomonas syringae pv. tomato (Pst) DC3000, two distinct nonhost bacterial pathogens. It also suppressed the HR triggered by transient coexpression of potato R3a and Phytophthora infestans Avr3a genes but did not compromise the HR triggered by recognition of Cladosporium fulvum AVR4 by tomato Cf- 4 . The ease of silencing in $N$. benthamiana makes it suitable for large scale VIGS experiments. A study of 192 cDNA-AFLP fragments, expressed during the HR following recognition of Avr4 from C. fulvum by tomato $C f-4$, was conducted in $N$. benthamiana and identified 15 Avr4-responsive tomato (ART) fragments that, when silenced, resulted in a compromised HR induced by both Avr4 in Cf-4 transgenic plants and the Inf1 gene from $P$. infestans [57]. In addition, silencing of HSP90, a nuclear GTPase, an L19 ribosomal protein, and a nucleotide bindingleucine rich repeat (NB-LRR)-type protein suppressed the HR [57]. Interestingly, silencing of the NB-LRR-type protein NRC1 not only affected the Cf-4/Avr4-induced HR and compromised Cf-4-mediated resistance to C. fulvum, but also revealed that this protein is required for the HR induced by the $R$ proteins Cf- 9 , LeEix, Pto, Rx, and Mi [58]. 


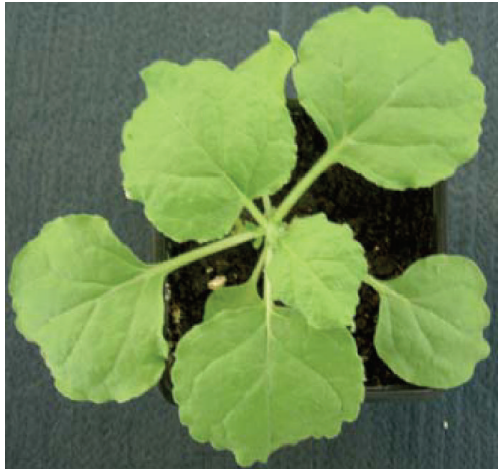

(a)

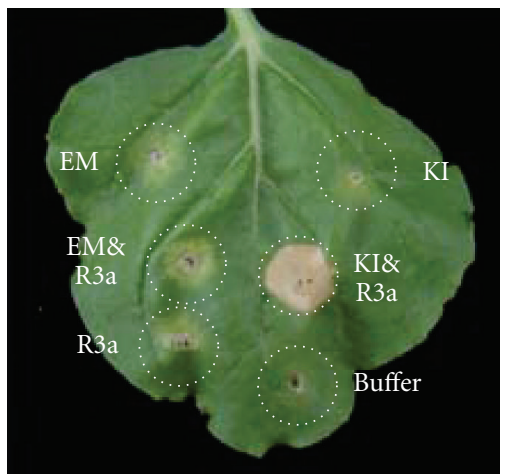

(d)

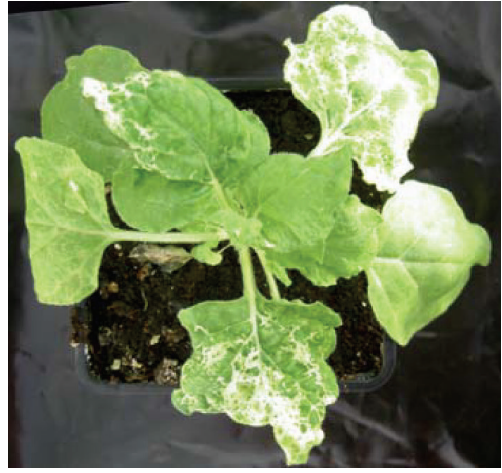

(b)

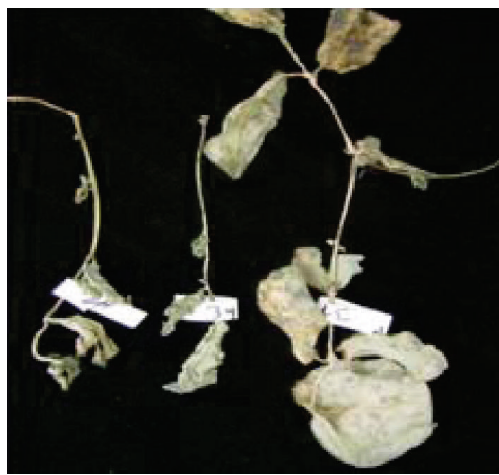

(e)

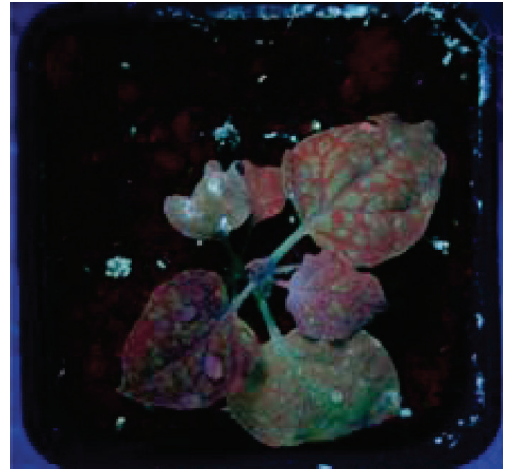

(c)

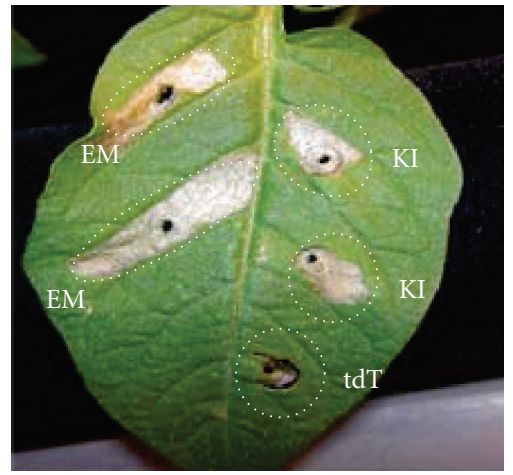

(f)

FIGURE 1: N. benthamiana plants, noninoculated (a), inoculated with pGR106::PDS to silence endogenous phytoene desaturase resulting in photo bleaching of leaves (b) and overexpressing GFP via a pGR106::GFP construct-viewed under UV light to show expression of GFP (c). Overexpression and coinfiltration of virulent Avr3a KI and avirulent Avr3a EM with the potato $R$ gene R3a are shown in (d). Naturally occurring PVX resistance in S. papita (e) and the recognition of virulent Avr3a KI and avirulent Avr3a EM alleles but not from tdT, used as a control, in S. chacoense (f).

A recently developed TRV RNA2 vector, which utilizes ligation-independent cloning (LIC), has been employed to assess the function of 400 tomato ESTs in N. benthamiana [59]. The function of SIMADS1 and its N. benthamiana homologous sequences, NbMADS4-1 and -2, was shown during flowering and demonstrated that NbMADS4-1 and NbMADS4-2 act nonredundantly in floral development. Silencing of either gene resulted in loss of organ identity. These studies show the potential for use of N. benthamiana as a "proxy" species for high-throughput gene function analysis for potato and other Solanaceae.

\subsection{Virus and Agrobacterium tumefaciens-based overexpression}

In addition to their role in VIGS, virus vectors can be used for overexpressing genes in plants. The AgrobacteriumPVXbased binary vector pGR106, an efficient silencing vector for Solanum species, can also be used for overexpressing genes, as shown for GFP in Figure $1[51,52]$. The search for novel sources of plant resistance, driven by knowledge of pathogen "effectors" with avirulent activities, rather than more traditional plant disease resistance breeding, has been coined "effectoromics" [60]. For example, overexpression of
$P$. infestans effectors in potato represents an opportunity to seek vital and invariant components of the P. infestans pathogenicity apparatus that can be targeted for sustainable potato protection. Information emerging from effectoromic studies will be useful to identify the cognate host $R$ genes as sources of durable disease resistance and to develop novel control strategies that are intrinsically difficult for the pathogen to overcome. The discovery of a conserved motif, RxLR, within many avirulence genes [61, 62] that is required for translocation of the effectors from pathogen haustoria into the plant cell [63] has had a tremendous impact on the prediction of pathogen effectors. Overexpression via pGR106 in N. benthamiana of 63 predicted $P$. infestans extracellular proteins (Pex) led to the discovery of two novel necrosis-inducing cDNAs, encoding extracellular proteins belonging to a large and complex protein family in Phytophthora [64]. Similarly, the recognition of the $P$. infestans effector $A v r 3 a$ by the potato $R$ gene $R 3 a$ [26] could be demonstrated in $N$. benthamiana [61]. Coinfiltration of $N$. benthamiana leaves with an $A$. tumefaciens strain carrying a construct expressing $R 3 a$ and a strain carrying a construct expressing the truncated avirulent Avr3a (Avr3a KI) sequence via PVX resulted in a confluent cell death response, not observed when overexpressing the truncated 
virulent $A v r 3 a$ ( $A v r 3 a$ EM) sequence (Figure 1). Using the $P$. infestans elicitins INF1, INF2A, and INF2B, the same PVX system has been adapted and optimized to screen Solanum plants for response to pathogen elicitors [65]. Of 31 potato species tested, 11 clones of Solanum huancabambense and Solanum microdontum responded with HR-like symptoms, which were also observed following infiltration with purified recombinant INF1, INF2A, and INF2B.

Two similar studies have been reported that utilize the two Avr3a alleles described above to identify potentially novel resistance mechanisms within wild potato accessions $[66,67]$. One study [66] utilized PVX to express the different Avr3a alleles in wild Solanum species, whereas the other [67] utilized Agrobacterium-only-based expression of the Avr $3 a$ alleles to circumvent the relative high level of resistance against PVX within the wild species tested (Figure 1). These studies identified similar sets of species that recognize both the EM and KI forms of AVR3a (unpublished data).

\section{WHERE NEXT FOR POTATO?}

Potato has entered an exciting new era, whereby the development of extensive genetic and genomic resources have opened up many new possibilities for studying important potato traits relevant to potato agronomy. Concomitant development of similar resources for other Solanaceous species, notably tomato, and a growing cohesiveness of the Solanaceae research community, as demonstrated by the "SOL vision" (http://www.sgn.cornell.edu/solanaceaeproject/) bode well for future genomic research of potato and its close relatives. Development of biotechnological tools for assaying potato gene function is likely to progress rapidly in the coming years.

\section{ACKNOWLEDGMENTS}

The authors would like to acknowledge the financial support of the Scottish Government Rural and Environment Research and Analysis Directorate, and the European Union.

\section{REFERENCES}

[1] C. Gebhardt, "Molecular markers, maps, and population genetics," in Potato Biology and Biotechnology: Advances and Perspectives, D. Vreugdenhil, Ed., chapter 5, pp. 77-89, Elsevier, Amsterdam, The Netherlands, 2007.

[2] H. van Os, S. Andrzejewski, E. Bakker, et al., "Construction of a 10,000-marker ultradense genetic recombination map of potato: providing a framework for accelerated gene isolation and a genomewide physical map," Genetics, vol. 173, no. 2, pp. 1075-1087, 2006.

[3] C. A. Hackett, B. Pande, and G. J. Bryan, "Constructing linkage maps in autotetraploid species using simulated annealing," Theoretical and Applied Genetics, vol. 106, no. 6, pp. 11071115, 2003.

[4] J. E. Bradshaw, B. Pande, G. J. Bryan, et al., "Interval mapping of quantitative trait loci for resistance to late blight [Phytophthora infestans (Mont.) de bary], height and maturity in a tetraploid population of potato (Solanum tuberosum subsp. tuberosum)," Genetics, vol. 168, no. 2, pp. 983-995, 2004.

[5] I. Simko, S. Jansky, S. Stephenson, and D. Spooner, "Genetics of resistance to pests and disease," in Potato Biology and Biotechnology: Advances and Perspectives, D. Vreugdenhil, Ed., chapter 7, pp. 117-155, Elsevier, Amsterdam, The Netherlands, 2007.

[6] H. J. van Eck, "Genetics of morphological and tuber traits," in Potato Biology and Biotechnology: Advances and Perspectives, D. Vreugdenhil, Ed., pp. 91-115, Elsevier, Amsterdam, The Netherlands, 2007.

[7] K. Arumuganathan and E. D. Earle, "Nuclear DNA content of some important plant species," Plant Molecular Biology Reporter, vol. 9, no. 4, p. 415, 1991.

[8] T. Gavrilenko, "Potato cytogenetics," in Potato Biology and Biotechnology: Advances and Perspectives, D. Vreugdenhil, Ed., chapter 10, pp. 203-216, Elsevier, Oxford, UK, 2007.

[9] S. D. Tanksley, M. W. Ganal, J. P. Prince, et al., "High density molecular linkage maps of the tomato and potato genomes," Genetics, vol. 132, no. 4, pp. 1141-1160, 1992.

[10] D. Zamir and S. D. Tanksley, "Tomato genome is comprised largely of fast-evolving, low copy-number sequences," Molecular \& General Genetics, vol. 213, no. 2-3, pp. 254-261, 1988.

[11] G. Schweizer, N. Borisjuk, L. Borisjuk, et al., "Molecular analysis of highly repeated genome fractions in Solanum and their use as markers for the characterization of species and cultivars," Theoretical and Applied Genetics, vol. 85, no. 6-7, pp. 801-808, 1993.

[12] Y. Wang, R. S. van der Hoeven, R. Nielsen, L. A. Mueller, and S. D. Tanksley, "Characteristics of the tomato nuclear genome as determined by sequencing undermethylated EcoRI digested fragments," Theoretical and Applied Genetics, vol. 112, no. 1, pp. 72-84, 2005.

[13] W. Zhu, S. Ouyang, M. Iovene, et al., "Analysis of $90 \mathrm{Mb}$ of the potato genome reveals conservation of gene structures and order with tomato but divergence in repetitive sequence composition," BMC Genomics, vol. 9, article 286, pp. 1-14, 2008.

[14] M. Crookshanks, J. Emmersen, K. G. Welinder, and K. L. Nielsen, "The potato tuber transcriptome: analysis of 6077 expressed sequence tags," FEBS Letters, vol. 506, no. 2, pp. 123126, 2001.

[15] C. M. Ronning, S. S. Stegalkina, R. A. Ascenzi, et al., "Comparative analyses of potato expressed sequence tag libraries," Plant Physiology, vol. 131, no. 2, pp. 419-429, 2003.

[16] B. Flinn, C. Rothwell, R. Griffiths, et al., "Potato expressed sequence tag generation and analysis using standard and unique cDNA libraries," Plant Molecular Biology, vol. 59, no. 3, pp. 407-433, 2005.

[17] J. Tang, B. Vosman, R. E. Voorrips, C. G. van der Linden, and J. A. M. Leunissen, "QualitySNP: a pipeline for detecting single nucleotide polymorphisms and insertions/deletions in EST data from diploid and polyploid species," BMC Bioinformatics, vol. 7, article 438, pp. 1-15, 2006.

[18] K. Kanyuka, A. Bendahmane, J. N. A. M. R. van der Voort, E. A. G. van der Vossen, and D. C. Baulcombe, "Mapping of intra-locus duplications and introgressed DNA: aids to map-based cloning of genes from complex genomes illustrated by physical analysis of the Rx locus in tetraploid potato," Theoretical and Applied Genetics, vol. 98, no. 5, pp. 679-689, 1999. 
[19] J. Song, F. Dong, and J. Jiang, "Construction of a bacterial artificial chromosome (BAC) library for potato molecular cytogenetics research," Genome, vol. 43, no. 1, pp. 199-204, 2000.

[20] Q. Chen, S. Sun, Q. Ye, S. McCuine, E. Huff, and H.-B. Zhang, "Construction of two BAC libraries from the wild Mexican diploid potato, Solanum pinnatisectum, and the identification of clones near the late blight and Colorado potato beetle resistance loci," Theoretical and Applied Genetics, vol. 108, no. 6, pp. 1002-1009, 2004.

[21] H. Kuang, F. Wei, M. R. Marano, et al., "The R1 resistance gene cluster contains three groups of independently evolving, type I R1 homologues and shows substantial structural variation among haplotypes of Solanum demissum," The Plant Journal, vol. 44, no. 1, pp. 37-51, 2005.

[22] F. Dong, J. Song, S. K. Naess, J. P. Helgeson, C. Gebhardt, and J. Jiang, "Development and applications of a set of chromosome-specific cytogenetic DNA markers in potato," Theoretical and Applied Genetics, vol. 101, no. 7, pp. 1001$1007,2000$.

[23] A. Bendahmane, K. Kanyuka, and D. C. Baulcombe, "The Rx gene from potato controls separate virus resistance and cell death responses," Plant Cell, vol. 11, no. 5, pp. 781-791, 1999.

[24] E. A. G. van der Vossen, J. N. A. M. R. van der Voort, K. Kanyuka, et al., "Homologues of a single resistance-gene cluster in potato confer resistance to distinct pathogens: a virus and a nematode," The Plant Journal, vol. 23, no. 5, pp. 567-576, 2000.

[25] S. Huang, V. G. A. A. Vleeshouwers, J. S. Werij, et al., "The R3 resistance to Phytophthora infestans in potato is conferred by two closely linked R genes with distinct specificities," Molecular Plant-Microbe Interactions, vol. 17, no. 4, pp. 428435, 2004.

[26] S. Huang, E. A. G. van der Vossen, H. Kuang, et al., "Comparative genomics enabled the isolation of the R3a late blight resistance gene in potato," The Plant Journal, vol. 42, no. 2, pp. 251-261, 2005.

[27] T.-H. Park, J. Gros, A. Sikkema, et al., "The late blight resistance locus Rpi-blb3 from Solanum bulbocastanum belongs to a major late blight R gene cluster on chromosome 4 of potato," Molecular Plant-Microbe Interactions, vol. 18, no. 7, pp. 722729, 2005.

[28] T. H. Park, V. G. A. A. Vleeshouwers, D. J. Huigen, E. A.G. van der Vossen, H. J. van Eck, and R. G. F. Visser, "Characterization and high-resolution mapping of a late blight resistance locus similar to R2 in potato," Theoretical and Applied Genetics, vol. 111, no. 3, pp. 591-597, 2005.

[29] I. Hein, K. McLean, B. Chalhoub, and G. J. Bryan, "Generation and screening of a BAC library from a diploid potato clone to unravel durable late blight resistance on linkage group IV," International Journal of Plant Genomics, vol. 2007, Article ID 51421, 5 pages, 2007.

[30] S. Pflieger, V. Lefebvre, and M. Causse, "The candidate gene approach in plant genetics: a review," Molecular Breeding, vol. 7, no. 4, pp. 275-291, 2001.

[31] J. Paal, H. Henselewski, J. Muth, et al., "Molecular cloning of the potato Gro1-4 gene conferring resistance to pathotype Ro1 of the root cyst nematode Globodera rostochiensis, based on a candidate gene approach," The Plant Journal, vol. 38, no. 2, pp. 285-297, 2004.

[32] C. S. Jung, H. M. Griffiths, D. M. De Jong, S. Cheng, M. Bodis, and W. S. De Jong, "The potato $P$ locus codes for flavonoid 3', 5'-hydroxylase," Theoretical and Applied Genetics, vol. 110, no. 2, pp. 269-275, 2005.
[33] L. Li, J. Strahwald, H.-R. Hofferbert, et al., "DNA variation at the invertase locus invGE/GF is associated with tuber quality traits in populations of potato breeding clones," Genetics, vol. 170, no. 2, pp. 813-821, 2005.

[34] C. W. B. Bachem, R. S. van der Hoeven, S. M. de Bruijn, D. Vreugdenhil, M. Zabeau, and R. G. F. Visser, "Visualization of differential gene expression using a novel method of RNA fingerprinting based on AFLP: analysis of gene expression during potato tuber development," The Plant Journal, vol. 9, no. 5, pp. 745-753, 1996.

[35] C. Bachem, R. S. van der Hoeven, J. Lucker, et al., "Functional genomic analysis of potato tuber life-cycle," Potato Research, vol. 43, no. 4, pp. 297-312, 2000.

[36] B. Brugmans, A. F. del Carmen, C. W. B. Bachem, H. van Os, H. J. van Eck, and R. G. F. Visser, "A novel method for the construction of genome wide transcriptome maps," The Plant Journal, vol. 31, no. 2, pp. 211-222, 2002.

[37] L. M. Trindade, B. M. Horvath, R. van Berloo, and R. G. F. Visser, "Analysis of genes differentially expressed during potato tuber life cycle and isolation of their promoter regions," Plant Science, vol. 166, no. 2, pp. 423-433, 2004.

[38] E. Ritter, J. I. Ruiz de Galarreta, H. J. van Eck, and I. Sánchez, "Construction of a potato transcriptome map based on the cDNA-AFLP technique," Theoretical and Applied Genetics, vol. 116, no. 7, pp. 1003-1013, 2008.

[39] V. E. Velculescu, L. Zhang, B. Vogelstein, and K. W. Kinzler, "Serial analysis of gene expression," Science, vol. 270, no. 5235, pp. 484-487, 1995.

[40] S. Saha, A. B. Sparks, C. Rago, et al., "Using the transcriptome to annotate the genome," Nature Biotechnology, vol. 20, no. 5, pp. 508-512, 2002.

[41] K. L. Nielsen, K. Grønkjær, K. G. Welinder, and J. Emmersen, "Global transcript profiling of potato tuber using LongSAGE," Plant Biotechnology Journal, vol. 3, no. 2, pp. 175-185, 2005.

[42] S. J. Emrich, W. B. Barbazuk, L. Li, and P. S. Schnable, "Gene discovery and annotation using LCM-454 transcriptome sequencing," Genome Research, vol. 17, no. 1, pp. 69-73, 2007.

[43] W. Rensink, A. Hart, J. Liu, S. Ouyang, V. Zismann, and C. R. Buell, "Analyzing the potato abiotic stress transcriptome using expressed sequence tags," Genome, vol. 48, no. 4, pp. 598-605, 2005.

[44] B. Kloosterman, O. Vorst, R. D. Hall, R. G. F. Visser, and C. W. Bachem, "Tuber on a chip: differential gene expression during potato tuber development," Plant Biotechnology Journal, vol. 3, no. 5, pp. 505-519, 2005.

[45] B. Kloosterman, D. De Koeyer, R. Griffiths, et al., "The potato transcriptome: a new look at transcriptional changes during tuber development using the POCI array," Functional \& Integrative Genomics, vol. 8, pp. 329-340, 2008.

[46] J. Muth, S. Hartje, R. M. Twyman, H.-R. Hofferbert, E. Tacke, and D. Prüfer, "Precision breeding for novel starch variants in potato," Plant Biotechnology Journal, vol. 6, no. 6, pp. 576-584, 2008.

[47] I. Simko, K. G. Haynes, and R. W. Jones, "Assessment of linkage disequilibrium in potato genome with single nucleotide polymorphism markers," Genetics, vol. 173, no. 4, pp. 22372245, 2006.

[48] R. Lu, A. M. Martín-Hernández, J. R. Peart, I. Malcuit, and D. C. Baulcombe, "Virus-induced gene silencing in plants," Methods, vol. 30, no. 4, pp. 296-303, 2003.

[49] O. Faivre-Rampant, E. M. Gilroy, K. Hrubikova, et al., "Potato virus X-induced gene silencing in leaves and tubers of potato," Plant Physiology, vol. 134, no. 4, pp. 1308-1316, 2004. 
[50] G. Brigneti, A. M. Martín-Hernández, H. Jin, et al., "Virusinduced gene silencing in Solanum species," The Plant Journal, vol. 39, no. 2, pp. 264-272, 2004.

[51] L. Jones, A. J. Hamilton, O. Voinnet, C. L. Thomas, A. J. Maule, and D. C. Baulcombe, "RNA-DNA interactions and DNA methylation in post-transcriptional gene silencing," Plant Cell, vol. 11, no. 12, pp. 2291-2302, 1999.

[52] R. Lu, I. Malcuit, P. Moffett, et al., "High throughput virusinduced gene silencing implicates heat shock protein 90 in plant disease resistance," The EMBO Journal, vol. 22, no. 21, pp. 5690-5699, 2003.

[53] C.-M. Ryu, A. Anand, L. Kang, and K. S. Mysore, "Agrodrench: a novel and effective agroinoculation method for virusinduced gene silencing in roots and diverse Solanaceous species," The Plant Journal, vol. 40, no. 2, pp. 322-331, 2004.

[54] M. M. Goodin, D. Zaitlin, R. A. Naidu, and S. A. Lommel, "Nicotiana benthamiana: its history and future as a model for plant-pathogen interactions," Molecular Plant-Microbe Interactions, vol. 21, no. 8, pp. 1015-1026, 2008.

[55] M. R. Godge, A. Purkayastha, I. Dasgupta, and P. P. Kumar, "Virus-induced gene silencing for functional analysis of selected genes," Plant Cell Reports, vol. 27, no. 2, pp. 209-219, 2008.

[56] E. M. Gilroy, I. Hein, R. van der Hoorn, et al., "Involvement of cathepsin B in the plant disease resistance hypersensitive response," The Plant Journal, vol. 52, no. 1, pp. 1-13, 2007.

[57] S. H. E. J. Gabriëls, F. L. W. Takken, J. H. Vossen, et al., "cDNAAFLP combined with functional analysis reveals novel genes involved in the hypersensitive response," Molecular PlantMicrobe Interactions, vol. 19, no. 6, pp. 567-576, 2006.

[58] S. H. E. J. Gabriëls, J. H. Vossen, S. K. Ekengren, et al., "An NB-LRR protein required for HR signalling mediated by both extra- and intracellular resistance proteins," The Plant Journal, vol. 50, no. 1, pp. 14-28, 2007.

[59] Y. Dong, T. M. Burch-Smith, Y. Liu, P. Mamillapalli, and S. P. Dinesh-Kumar, "A ligation-independent cloning tobacco rattle virus vector for high-throughput virus-induced gene silencing identifies roles for NbMADS4-1 and -2 in floral development," Plant Physiology, vol. 145, no. 4, pp. 1161-1170, 2007.

[60] S. Kamoun and S. B. Goodwin, "Fungal and oomycete genes galore," New Phytologist, vol. 174, no. 4, pp. 713-717, 2007.

[61] M. R. Armstrong, S. C. Whisson, L. Pritchard, et al., "An ancestral oomycete locus contains late blight avirulence gene Avr3a, encoding a protein that is recognized in the host cytoplasm," Proceedings of the National Academy of Sciences of the United States of America, vol. 102, no. 21, pp. 7766-7771, 2005.

[62] A. P. Rehmany, A. Gordon, L. E. Rose, et al., "Differential recognition of highly divergent downy mildew avirulence gene alleles by RPP1 resistance genes from two Arabidopsis lines," Plant Cell, vol. 17, no. 6, pp. 1839-1850, 2005.

[63] S. C. Whisson, P. C. Boevink, L. Moleleki, et al., "A translocation signal for delivery of oomycete effector proteins into host plant cells," Nature, vol. 450, no. 7166, pp. 115-118, 2007.

[64] T. A. Torto, S. Li, A. Styer, et al., "EST mining and functional expression assays identify extracellular effector proteins from the plant pathogen Phytophthora," Genome Research, vol. 13, no. 7, pp. 1675-1685, 2003.

[65] V. G. A. A. Vleeshouwers, J.-D. Driesprong, L. G. Kamphuis, et al., "Agroinfection-based high-throughput screening reveals specific recognition of INF elicitins in Solanum," Molecular Plant Pathology, vol. 7, no. 6, pp. 499-510, 2006.
[66] N. Champouret, H. Rietman, J. I. Bos, et al., "Functional allele mining: a new approach to identify R-gene homologues in Solanum," in Proceedings of the 13th International Congress on Molecular Plant-Microbe Interactions, p. 177, Sorrento, Italy, July 2007.

[67] I. Hein, J. Squires, P. Birch, and G. J. Bryan, "Screening wild potato accessions for resistance to the virulent allele of the Phytophthora infestans effector Avr3a," in Proceedings of the 13th International Congress on Molecular Plant-Microbe Interactions, p. 259, Sorrento, Italy, July 2007. 

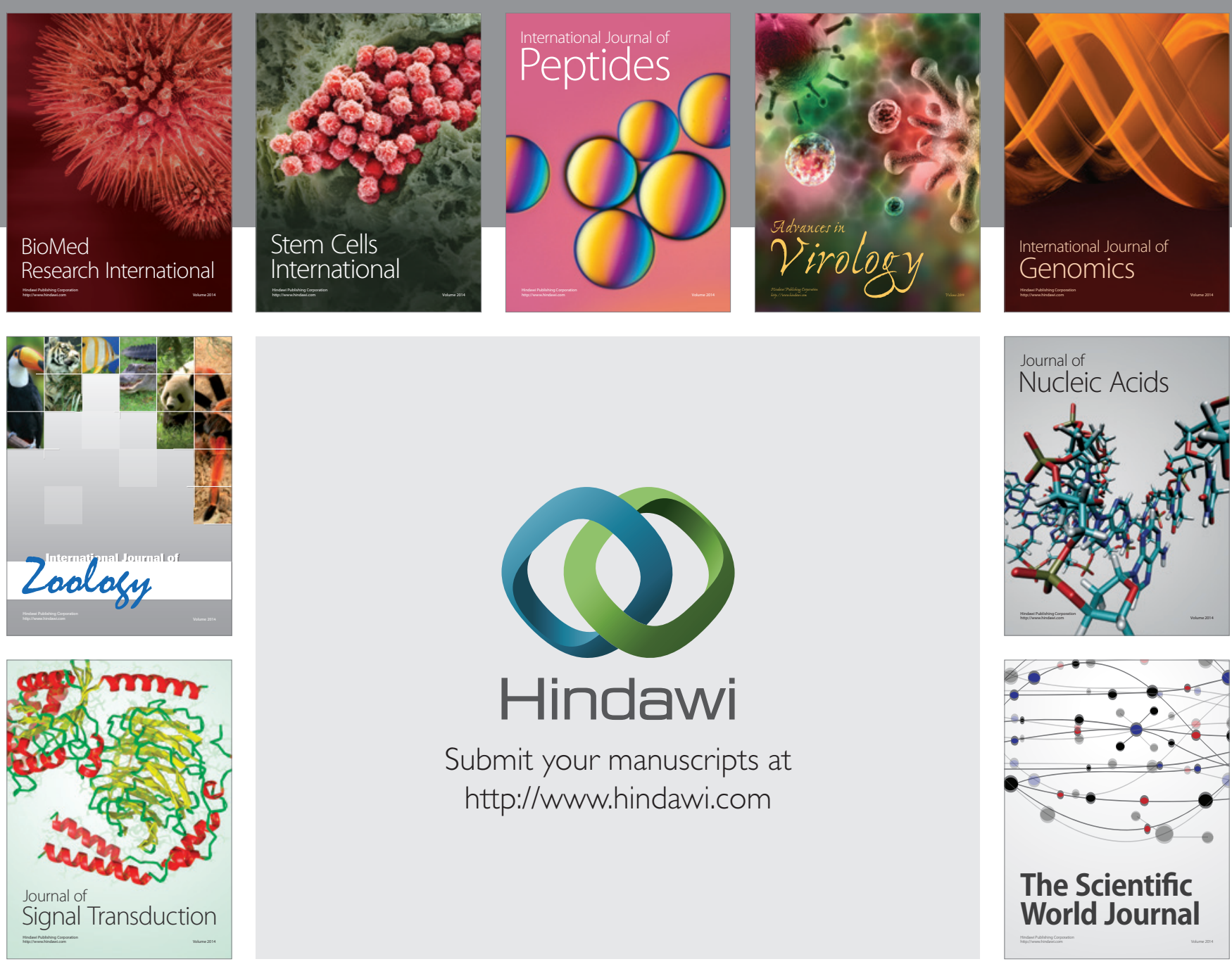

Submit your manuscripts at

http://www.hindawi.com
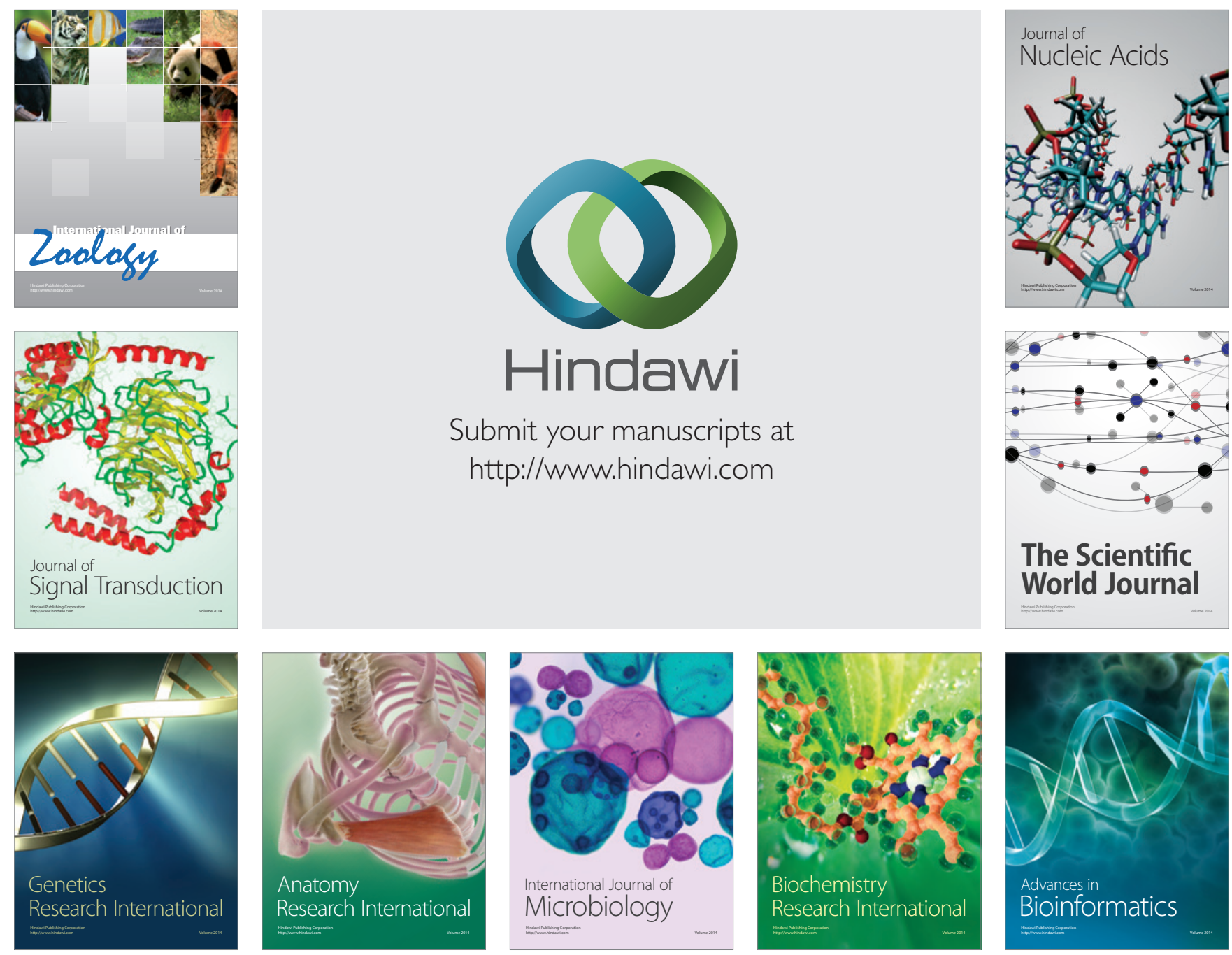

The Scientific World Journal
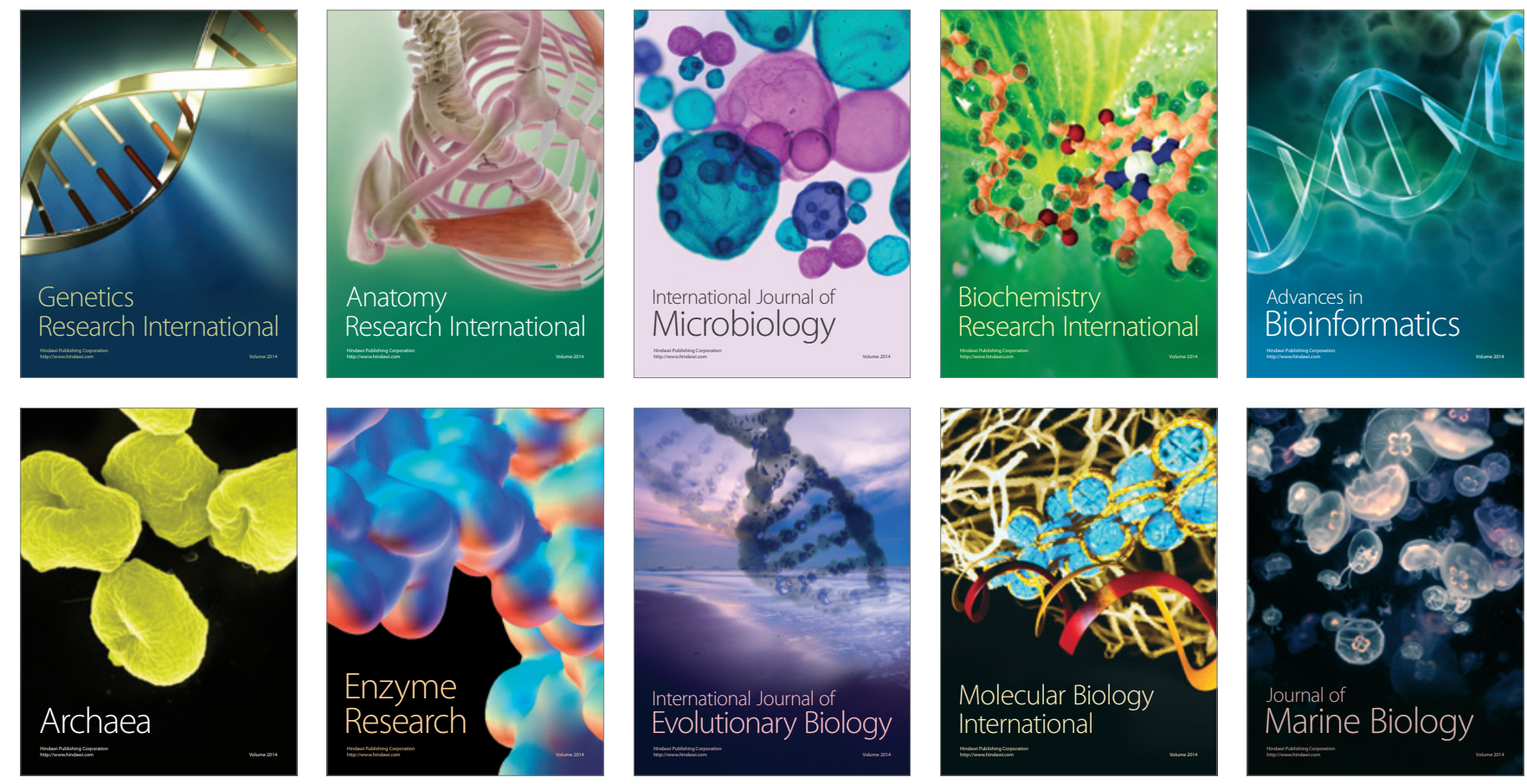